\title{
Predictors of stunting among pediatric children living with HIV/AIDS, Eastern Ethiopia
}

\author{
Dawit Gezahegn ${ }^{1}$, Gudina Egata ${ }^{2}$, Tesfaye Gobena ${ }^{3}$, Berhanu Abebaw ${ }^{4}$ \\ ${ }^{1}$ Nutrition expert at Hiwot Fana Specialized Hospital, Haramaya University, Ethiopia \\ ${ }^{2,3}$ Department of Public Health, College of Health and Medical Sciences, Haramaya University, Ethiopia \\ ${ }^{4}$ Department of Nutrition and Dietetics, School of Public Health, Bahir Dar University, Ethiopia
}

\section{Article Info}

Article history:

Received Feb 21, 2020

Revised Apr 23, 2020

Accepted May 4, 2020

\section{Keywords:}

Stunting

HIV/AIDS

Pediatric children

Eastern

\begin{abstract}
Globally, there were about 3.4 million pediatric children $(<15$ years of age) who were living with HIV/AIDS. Ethiopia has one of the highest rates of malnutrition in Sub-Saharan Africa. As of 2013, there were about 160,000 pediatric children living with HIV/AIDS in Ethiopia. Even though undernutrition makes it difficult to combat HIV/AIDS, there is paucity of information on the magnitude of stunting and its predictors among seropositive pediatric children in low-income countries like Ethiopia. Institution based quantitative cross sectional study design was employed on 414 randomly selected pediatric (5-15 years) children living with HIV/AIDS in Harari Region and Dire Dawa City Administration Public Hospitals, Eastern Ethiopia. Pretested interviewer administered questionnaire and patient card review was held to collect data. Data were entered through Epi-data and exported to SPSS for analysis. The WHO Anthros plus software was used to calculate the anthropometric indices. Bivariate and Multivariable analysis along with $95 \%$ CI were done to identify predictors of stunting. Level of statistical significance was declared at P-value $<0.05$. The prevalence of stunting was found to be $30.9 \%$ (95\%CI: 26.0-36.0\%). Rural residence [AOR=4.0, (95\%CI: 2.22, 7.17)], family monthly income of $\leq 500$ ETB [AOR $=5.79$, (95\%CI: 2.82, 11.60)], being anemic [AOR=3.17, $(95 \% \mathrm{CI}$ : $2.13,4.93$ )] and the presence of diarrhea [AOR=6.21, 95\% (CI: 3.39, 9.24)] were predictors of stunting. Thus, collaborative measures should be undertaken (to decrease frequent infections and to improve the economic status) to combat chronic malnutrition during HIV/AIDS treatment.
\end{abstract}

This is an open access article under the CC BY-SA license.

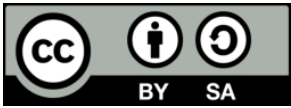

\section{Corresponding Author:}

Berhanu Abebaw,

Department of Nutrition and Dietetics,

School of Public Health,

Bahir Dar University, Ethiopia.

Email: birhanua20@gmail.com

\section{INTRODUCTION}

Malnutrition and HIV/AIDS worsen one another. Malnutrition contributes to immune system weakening, making the body vulnerable to frequent illness and increasing its energy and nutrient demand, there by accelerating the disease progression. In turn, HIV infection exacerbates malnutrition through its attacks on the immune system and its impact on the reduced food intake [1,2]. Nutritional status controls the immunological response to HIV infection, affecting the overall clinical outcomes [3]. Malnutrition further weakens the immune system, increasing susceptibility to opportunistic infections. Even on ART, there is a continuous need for PLHIV to consume a nutritious diet to maintain weight and prevent 
micronutrient deficiencies $[4,5]$. The links between malnutrition and HIV/AIDS increase the negative effects of HIV infection on human development at individual, household, community and national levels [6].

Nutrition is a critical component of HIV treatment, care, and support, as recommended by different international organizations (such as, WHO, UNAIDS, and others) which are working on HIV/AIDS. Nutritional support has a role within clinical and community services in case of commitment, adherence and retention in care and treatment of patients on ART [7, 8]. Clinical studies show that pediatric children ( $<15$ years) living with HIV/AIDS have poor nutritional status, mostly due to the presence of infections (diarrhea) and OIs like candidiasis, which can cause pain during swallowing, and further impedes normal eating patterns [9]. People living with HIV need to consume additional $30 \%$ calories than uninfected counterparts, making nutritional support a key component [10]. Undernutrition and its connection with food insecurity, poverty, and co-infections pose a serious challenge to efforts to combat HIV/AIDS by denying access to a nutrition-rich diet $[11,12]$. Undernutrition is a significant factor affecting HIV/AIDS care and treatment in resource limited settings [13, 14]. Individuals at each stages of HIV disease are at risk of developing undernutrition and their nutritional status is a strong predictor of the progression, survival rate and functional status of individuals [15]. Several studies have demonstrated the link between undernutrition and HIV/AIDS disease progression [16, 17]. Globally, there were about 3.4 million children ( $<15$ years of age) who were living with HIV/AIDS; and 340,000-450,000 new infections in this age group per year [18]. The greatest proportion ( $>90 \%$ ) of children who acquired HIV infection live in Sub-Saharan Africa [19].

Ethiopia has one of the highest rates of malnutrition in Sub-Saharan Africa, and faces acute and chronic malnutrition and micronutrient deficiencies [20]. As of 2013, there were about 160,000 children ( $<15$ years) living with HIV/AIDS in Ethiopia [21]. While rates of stunting have dropped in many areas of the country over the past decade, Ethiopia still faces a huge burden from chronic malnutrition with $37 \%$ of under five children stunted, of whom about $12 \%$ are severely stunted [22]. The high levels of stunted growth among pediatric children (living with HIV) have been reported in many developing countries. A study in Uganda revealed $36.2 \%$ of adolescents living with HIV/AIDS as stunted; of whom, $11.1 \%$ were severely stunted [23]. Another study conducted among HIV positive children at two referral hospitals in northwest Ethiopia, revealed the magnitude of malnutrition as 42.9\% [24]. Even though there are ample literatures regarding the nutritional status of different age groups of people, little emphasis is given and little is understood about the burden of malnutrition in pediatric children living with HIV/AIDS. Therefore, this study aimed to assess the predictors of stunting among pediatric children living with HIV/AIDS in eastern Ethiopia.

\section{RESEARCH METHOD}

\subsection{Study area and design}

Institution based cross sectional study design was employed among pediatric children in Harari region and Dire Dawa city administration, Ethiopia in February 2017. Harar town (Capital of Harari region) and Dire Dawa city administration are located in the eastern part of Ethiopia around 526 and 515-kilo meters from Addis Ababa respectively. Harari region had a total population of 183,344 (Ethiopian central statistics authority's 2007 report) and the total population in Dire Dawa city administration was 405,808 (unpublished city administration's 2006 Ethiopian calendar report). Harari region has 5 governmental hospitals, 2 private hospitals, 8 health centers, 33 higher and medium private clinics, and 31 health posts. Dire Dawa city administration has 2 government hospitals, 15 government health centers, 4 private hospitals and more than 10 private clinics. In Harari region, there were about 5814 HIV positive persons who were on HAART treatment and 183 of them were pediatric children who were on ART (Unpublished report of Harari regional health Bureau, 2015/16). Similarly, in Dire Dawa city administration there were about 9815 HIV positives and 440 of them were pediatric children who were on ART in public hospitals (Unpublished report of Dire Dawa health bureau, 2016). All pediatric age children (5-15 years) who were living with HIV/AIDS and were on follow-up at the ART clinics of the selected health facilities participated in the study. Children who could not stand due to physical deformities and children with incomplete registered data were excluded from the study.

\subsection{Sample size and sampling procedures}

After comparing the sample size for the first and second specific objectives (to take the larger sample size), the sample size was finally determined using a formula for estimation of single population proportion, taking degree of precision 5\%, magnitude of undernutrition among HIV positive children as $42.9 \%$ [24] and 10\% non-response rate. Accordingly, the sample size was estimated to be 414. In Harari region there are two public hospitals (Hiwot Fana specialized university hospital and Jugala hospital). Similarly, in Dire Dawa city administration, there are two public hospitals (Dile Chora hospital and Sabian 
hospital). By taking the total number of pediatric children (5-15 years) who were attending ART clinic in those four hospitals as 623, the sample size was proportionally allocated to each hospital. Then, study participants were selected using the simple random sampling technique (considering the registration book as a good source of the sampling frame).

\subsection{Data collection tool and procedures}

Data were collected using a pretested structured questionnaire. The tool was developed by reviewing various literatures and efforts were made to include important predictors. The questionnaire was initially developed in English and translated into local language spoken in the study area ("Amharic, Afan Oromo, and Somali"). Then it was retranslated back to English language. Comparison was made to check for its consistency. Data were collected using face-to-face interview and review of medical records. Four female diploma graduate Nurses who were working in health facilities other than the selected public health facilities did the interview and chart review. Pediatric children (5-15 years) medical record charts were obtained and lists of children were provided to ART care provider to inform the data collectors, when they come to the hospital during the study period. Since pediatric children (5-15 years) living with HIV/AIDS were appointed every two weeks for follow up, all children were accessed in a month (the data collection period). When the health care provider referred the patient to the data collectors, the collectors informed the study participants and parents (caretakers) about the purpose of the study and both written informed consent from the caretakers and assent from the children were taken. Then after, interview was done in private room in the facility. The study ensured individual information will not be disclosed and will be kept confidential.

\subsection{Data quality management}

Two days training was given for all data collectors and discussion was made on the objective of the study, familiarization on data collection tool and each variable on the questionnaire and its implication. There was demonstration and practical session on interviewing and anthropometric measurements. The questionnaire was pretested at Haramaya hospital (not part of the study area), on 5\% of the total sample size and after the pretest, all necessary adjustments were made. Relative technical error of measurement (percentage TEM) was calculated to minimize anthropometric measurement errors. Data collectors' accuracy of anthropometric measurements was standardized with their trainer during training and pretesting. During data collection, data collectors took at least two separate height measurements for an individual and repeat when the variation of the two measures is greater than 0.1 centimeter. The anthropometric measurements were taken by following standard anthropometric techniques [25]. During the actual data collection, the supervisors made close supervision. The collected data were cross checked on each day of activity for consistency and completeness. Then the data were double entered into Epi data software with two data clerks independently and consistency between the two data sets was checked. Then mismatched data were crosschecked with the hard copy and corrected accordingly.

\subsection{Anthropometric measurements}

The anthropometric data were collected using the procedure stipulated by the WHO (2006) for taking anthropometric measurements. Before taking anthropometric data for children, first their age was determined in order to ensure the target population. All pediatric children aged 5-15 years height was measured using a stadiometer with the nearest 0.1 centimeter. Height was measured with subjects standing straight on a smoothly flat surface with their heels together, eyes straight forward, and touching the standing board at the heels, buttocks and the back of the head. For a child, two separate measurements were collected and the average values were used for analysis. Z-score was determined using the Anthro-Plus software. Based on the Z-score, a child was considered as stunted or not. A child was considered as being stunted when the height-for-age Z score was $<-2$ SD.

\subsection{Data processing and analysis}

After the data were checked for completeness and consistency, it was entered through Epi data version 3.1 software. Then after, the data were exported to SPSS version 22 for analysis. For anthropometric variables, WHO Anthro plus software was used and after the Z-score was calculated, it was exported to SPSS for further analysis. Descriptive analysis, including frequency and cross tabulations were done to describe the characteristics of study participants. Dietary diversity was calculated and the mean score was used to classify the adequacy of nutrient intake [26, 27]. The household food insecurity access scale (HFIAS) categories were calculated and households were categorized as food secured or not [28]. Data of the study subjects' were expressed as means \pm SD. Bivariate analysis was done to identify candidate variables for multivariable logistic regression then those variables having a p-value $<0.2$ were entered in multivariable logistic regression model. The outcome variable was stunting. Results were reported by using Odds Ratio and 95\% CI. P-value less than 0.05 indicated a statistically significant association.

Int. J. Public Health Sci, Vol. 9, No. 2, June 2020: 82 - 89 


\section{RESULTS AND DISCUSSION}

\subsection{Socio-demographic characteristics}

A total of 405 Pediatric children (5-15 years) patients attending ART clinic at public hospitals of Harari town and Dire Dawa City Administration eastern Ethiopia were participated making a response rate of $97 \%$. More than half $(54.6 \%)$ were females and $263(64.9 \%)$ of the participants were in the age group of $10-15$, with a mean age of 10.62 ( \pm 2.3 years). One hundred ninety-two (47.4\%) participants were Orthodox Christianity followers. Regarding the ethnicity, more than half $(52.2 \%)$ were from Oromo ethnic group. Three hundred $(74.1 \%)$ were resided in urban areas. Regarding the parental status of the children, 235(58.0\%) children have both parents (mother and father), and 74(18.3\%) have neither mother nor father alive. The majority (91.4\%) of study participants have caregiver. Two hundred thirty four $(57.8 \%)$ of the families have average monthly income of $\geq 1000$ ETB and $15.8 \%$ have average monthly income of $\leq 500$ ETB that can be seen in Table 1 .

Table 1. Socio-demographic characteristics of pediatric children living with HIV/ADIS

\begin{tabular}{cccc}
\hline Variables & Category & Frequency & Percentage \\
\hline Age (years) & $5-9$ & 142 & 35.1 \\
Sex & $10-15$ & 263 & 64.9 \\
& Male & 184 & 45.4 \\
Religion & Female & 221 & 54.6 \\
& Orthodox & 192 & 47.4 \\
& Muslim & 138 & 34.1 \\
& Protestant & 74 & 18.3 \\
Ethnicity & Catholic & 1 & 0.2 \\
& Oromo & 214 & 52.8 \\
Residence & Amhara & 145 & 35.8 \\
& Harari & 17 & 4.2 \\
Parental status & Somali & 5 & 1.2 \\
& Tigre & 17 & 4.2 \\
Fatrine availability & Other $¥$ & 7 & 1.7 \\
Presence of caregiver & Rural & 105 & 25.9 \\
& Urban & 300 & 74.1 \\
Caregiver & Both parents (Mother and Father) & 235 & 58.0 \\
& Only one parent & 96 & 23.7 \\
& Both absent & 74 & 18.3 \\
& Yes & 370 & 91.4
\end{tabular}

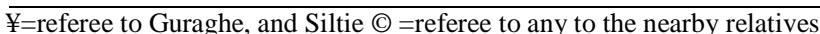

\subsection{Food security status and dietary practice of participants}

Nearly two third (66.4\%) of the respondents' households were food insecured and 384(94.8\%) have good dietary diversity score that can be seen in Figure 1.

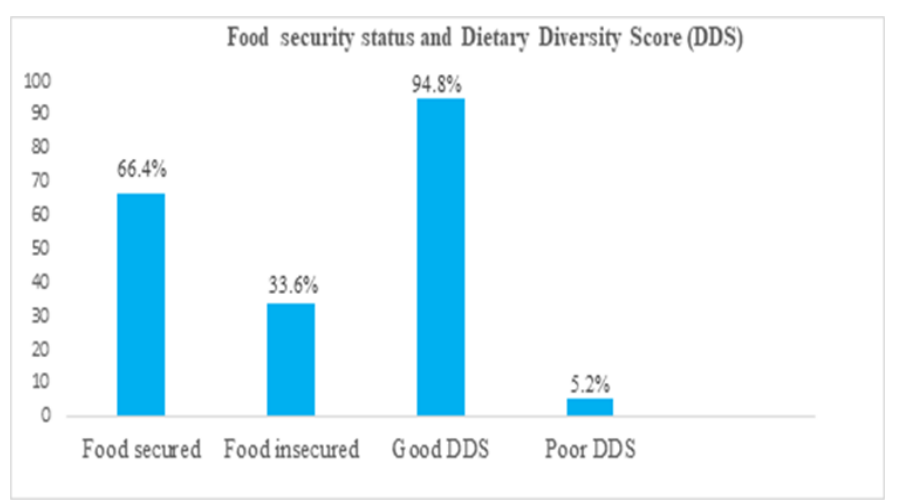

Figure 1. Household food security status and DDS among children living with HIV/AIDS 


\subsection{Medical characteristics}

Only less than one fourth (22.7\%) of the participants had therapeutic food during the course of ART treatment and $247(61.0 \%)$ had no history of dietary counseling. More than one third (34.3\%) had eating problems and 110(27.2\%) reported loss of appetite. Only sixty one (15.1\%) of the respondents had any of the opportunistic infections, of which nearly half $(46.7 \%)$ was pneumonia. Most of study participants (93.8\%) were in the WHO clinical stage one. One hundred eighty four (45.4\%) had $\geq 7$ years' duration of ART follow up. Eighty nine (22.0\%) were anemic. About 166(41\%) and 345(85.1\%) had baseline and current CD4 counts of $\geq 500$ respectively. Three hundred fifty-four $(87.4 \%)$ of the children had good adherence of the drug and $369(91.1 \%)$ had no history of diarrhea in the past two weeks that can be seen in Table 2.

Table 2. Medical characteristics among pediatric children living with HIV/ADIS

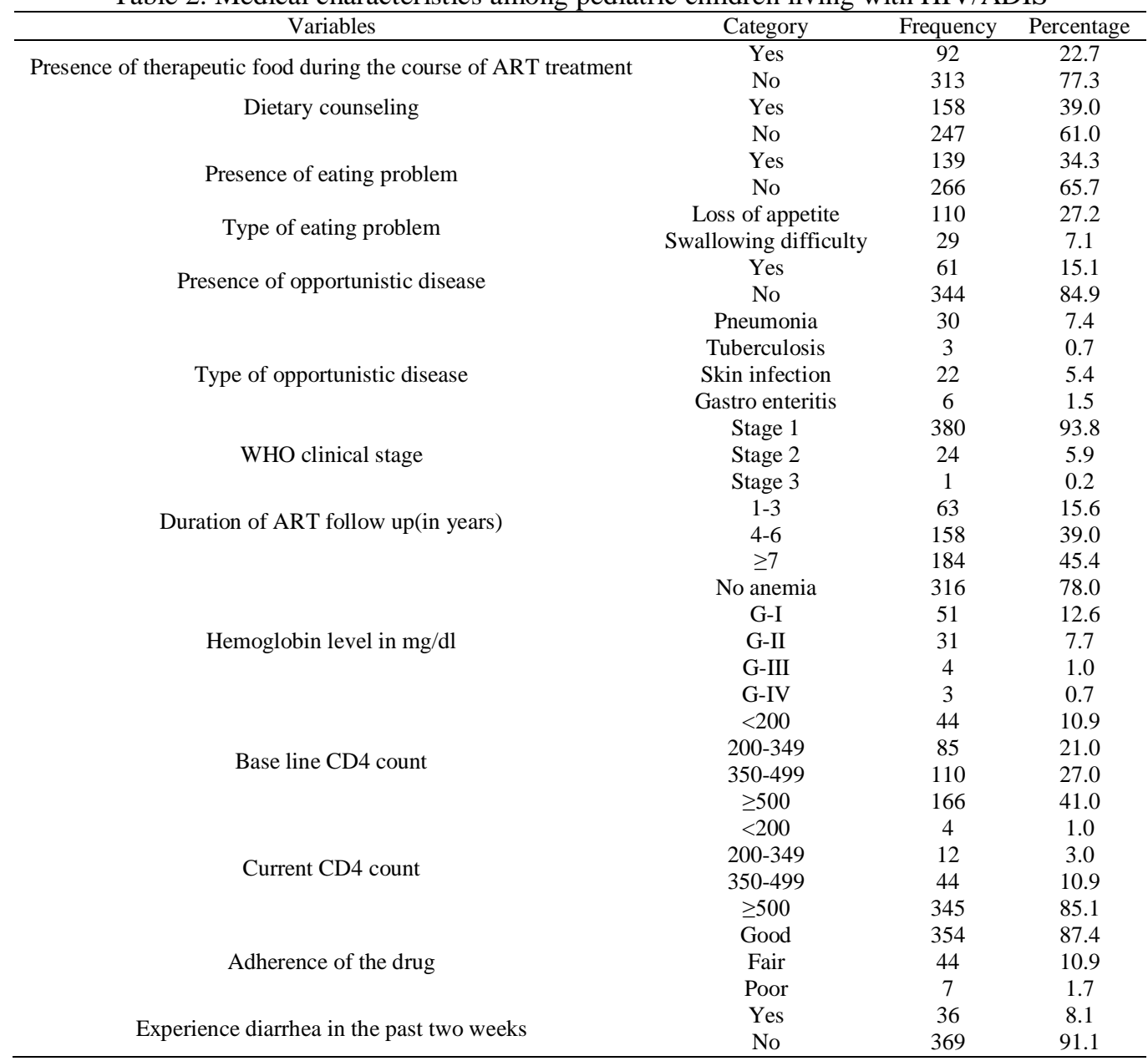

\subsection{Magnitude of Stunting among pediatric children living with HIV/ADIS}

About $30.9 \%, 95 \%$ CI $(26.0 \%, 36.0 \%)$ of the respondents were stunted (HAZ) that can be seen in Figure 2.

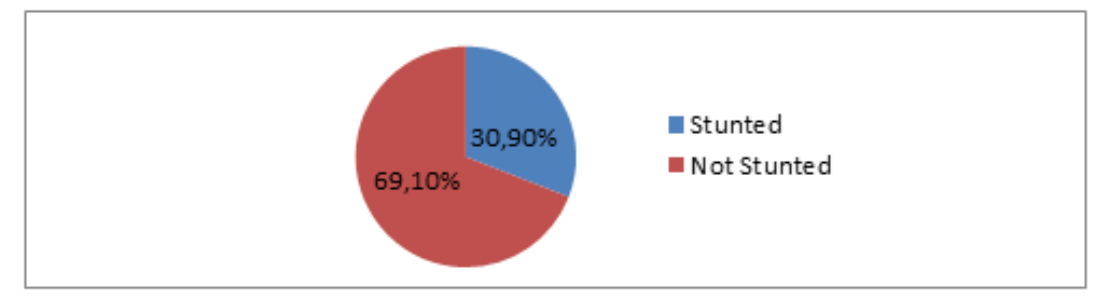

Figure 2. Magnitude of stunting among pediatric children living with HIV/AIDS

Int. J. Public Health Sci, Vol. 9, No. 2, June 2020: 82 - 89 


\subsection{Predictors of Stunting among pediatric children living with HIV/AIDS}

From the various explanatory variables rural residence, family monthly income of $\leq 500$ ETB, benig anemia, presence of diarrhea, duration of ART treatment follow-up, follow-up interval of ART treatment were found to had a p-value of $<0.2$ (in the Bivariate analysis) and were a candidate for the multivariate logistic regression. Accordingly, being rural resident, having family monthly income of $\leq 500$ ETB, being anaemic and having diarrhea were found to predictors of stunting. Children who resided in rural area were four times $[\mathrm{AOR}=4.00,95 \% \mathrm{CI}(2.22,7.17)]$ more likely to be stunted than those children who were urban residents. Those children who had an average family monthly income of $\leq 500$ ETB were 5.79 times $[\mathrm{AOR}=5.79,95 \% \mathrm{CI}(2.82,11.60)]$ more likely to be stunted compared to those who had an income of $\geq 1000$ ETB. Children who were anemic were 3.17 times [AOR=3.17, 95\%CI $(2.13,4.93)$ more likely to be stunted than those children who had no anemia. Those children who had diarrhea in the past two weeks were 6.21 times $[\mathrm{AOR}=6.21,95 \% \mathrm{CI}(3.39,9.24)]$ more likely to be stunted than those children hadn't diarrhea that can be seen in Table 3 .

Table 3. Binary logistic regression that shows the association of variables with stunting, among Pediatric children living with HIV/ADIS

\begin{tabular}{|c|c|c|c|c|}
\hline \multirow{2}{*}{ Variables } & \multicolumn{2}{|c|}{ Stunting } & \multirow{2}{*}{$\operatorname{COR}(95 \% \mathrm{CI})$} & \multirow{2}{*}{$\operatorname{AOR}(95 \% \mathrm{CI})$} \\
\hline & Yes $(\%)$ & No $(\%)$ & & \\
\hline \multicolumn{5}{|l|}{ Age (in years) } \\
\hline $5-9$ & $67(36.8)$ & $115(63.2)$ & $1.65(1.08,2.53)$ & $1.64(2.45,7.96)$ \\
\hline $10-15$ & $58(26.0)$ & $165(74.0)$ & 1 & 1 \\
\hline \multicolumn{5}{|l|}{ Residence } \\
\hline Rural & $59(43.8)$ & $46(56.2)$ & $4.54(2.83,7.29)$ & $4.0(2.22,7.17)^{*}$ \\
\hline Urban & $66(22.0)$ & $234(78.0)$ & 1 & 1 \\
\hline \multicolumn{5}{|l|}{ Family income } \\
\hline$\leq 500$ Birr & $49(48.0)$ & $53(52.0)$ & $3.49(2.08,5.87)$ & $5.79(2.82,11.60)^{*}$ \\
\hline 501-999 Birr & $35(32.7)$ & $72(67.3)$ & $1.84(1.08,3.12)$ & $3.00(1.42,6.34)$ \\
\hline$\geq 1000$ Birr & $41(20.9)$ & $155(79.1)$ & 1 & 1 \\
\hline \multicolumn{5}{|l|}{ Dietary counseling } \\
\hline Yes & $37(23.4)$ & 121(76.6) & 1 & 1 \\
\hline No & $88(35.6)$ & $159(64.4)$ & $1.17(0.77,1.79)$ & $1.79(0.46,1.37)$ \\
\hline \multicolumn{5}{|c|}{ Presence of eating problem } \\
\hline Yes & $45(32.4)$ & $94(67.6)$ & $1.11(0.72,1.73)$ & $1.07(0.60,1.90)$ \\
\hline No & $80(30.1)$ & $186(69.9)$ & 1 & 1 \\
\hline \multicolumn{5}{|c|}{ Duration of ART treatment follow up } \\
\hline $1-3$ years & $17(27.0)$ & $46(73.0)$ & $0.55(0.29,1.03)$ & $1.23(0.05,1.18)$ \\
\hline 4-6 years & $34(21.5)$ & $124(78.5)$ & $1.41(0.25,0.66)$ & $0.39(0.17,1.91)$ \\
\hline$\geq 7$ years & $74(40.2)$ & $110(59.8)$ & 1 & 1 \\
\hline \multicolumn{5}{|c|}{ Hemoglobin level in mg/dl } \\
\hline Anemic & $36(40.4)$ & $53(59.6)$ & $1.94(1.20,3.15)$ & $3.17(2.13,4.93)^{*}$ \\
\hline Non Anemic & $89(28.2)$ & $227(71.8)$ & 1 & 1 \\
\hline \multicolumn{5}{|l|}{ Diarrhea } \\
\hline Yes & $14(38.9)$ & $22(61.1)$ & $8.63(4.92,15.15)$ & $6.21(3.39,9.24)^{*}$ \\
\hline No & $111(30.1)$ & $258(69.9)$ & 1 & 1 \\
\hline \multicolumn{5}{|l|}{ Food security status } \\
\hline Food secured & $86(32.0)$ & $183(68.0)$ & 1 & 1 \\
\hline Food insecured & $39(28.7)$ & $97(71.3)$ & $1.86(0.54,1.34)$ & $0.82(0.40,1.68)$ \\
\hline \multicolumn{5}{|l|}{ Dietary Diversity } \\
\hline$<4$ food groups & $5(23.8)$ & $16(76.2)$ & $0.69(0.25,1.92)$ & $1.01(0.29,3.54)$ \\
\hline$\geq 4$ food groups & $120(31.2)$ & $264(68.8)$ & 1 & 1 \\
\hline
\end{tabular}

$*=$ Statistically Significant $(\mathrm{P}-$ value $<0.05), \mathrm{CI}=$ Confidence Interval, COR $=$ Crude Odds Ratio, AOR = Adjusted Odds Ratio, ART=Antiretroviral Therapy

Ethiopia has prioritized nutrition as a critical component of HIV treatment, care, and support by setting guidelines and developing implementation manuals [29]. Even though efforts are being made, the magnitude of stunting in this study is found to be $30.9 \%$ (95\% CI: 26.0-36.0\%); which is higher than the studies done in Ethiopia (24.7\%) [30] and Nigeria (17.1\%) [31]. However, the magnitude is lower than the studies conducted in two referral hospitals in Northwest Ethiopia (65\%) [24], in Uganda (68\%) [32], and in Mozambique (57.4\%) [33]. These variations might be due to the difference in the study setting, study groups, sample size, and quality of care in the facility (including nutritional support).

In this study, children who had diarrhea in the past two weeks were 6.21 times more likely to be stunted compared to their counter parts. This finding is supported by a study done in Ethiopia [30], in which children who experienced diarrhea were 2.1 times more likely to be stunted. The probable reason is that frequent infections like diarrhea, increases nutrient loses and has also its own role in reducing food intake 
by decreasing appetite and eagerness to eat. The presence of anemia was also another predictor variable for stunting; those pediatric children who were anemic were 3.17 times more likely to be stunted compared to those children who had no anemia. This finding is supported by a study done in Ethiopia [30]. This is due to the fact that children with chronic diseases like HIV/AIDS have a compromised immunity; which can further predispose to different infections/diseases. On the other hand, lower family monthly income was another predictor variable; those pediatric children who had monthly family income of $\leq 500$ ETB were 5.79 times more likely to be stunted compared to those children whose family monthly income was $\geq 1000$ ETB. The finding is supported by a study done in Arba Minch, Ethiopia [34]. This could be possibly due to, children from the poor families (lower family income) will be at risk of getting enough food, which further predisposes to infection/illness, and ultimately contribute to the development of malnutrition. Rural residence was significantly associated with stunting; those children who resided in rural area were four times more likely to be stunted, compared to their counter parts. The finding is consistent with many other studies conducted in Ethiopia. The primary reason might be, since the study is conducted in the hospitals (found in urban areas), those children living with HIV/AIDS will suffer from travel costs, which will further exert its negative effect on follow ups and treatment adherence. Those children who resided in the rural areas probably have a decreased media coverage, which will lead them to have inadequate knowledge and awareness about the issue.

\title{
4. CONCLUSION
}

There is high level of stunting among children (living with HIV/AIDS) in the study area. Being rural resident, lower family monthly income $(\leq 500 \mathrm{ETB})$, being anemic and the presence of diarrhea were significantly associated with stunting. Thus, collaborative measures should be undertaken (to decrease frequent infections, thereby improving the quality of care), efforts should also be made to improve the economic status and ultimately, to combat chronic malnutrition during HIV/AIDS treatment.

\author{
ABBREVIATIONS \\ AIDS: Acquired Immune Deficiency syndrome \\ AOR: Adjusted Odds Ratio \\ CI: Confidence Interval \\ ETB: Ethiopian Birr \\ OIs: Opportunistic Infections \\ PLWHA: People Living With HIV/AIDS \\ ART=Antiretroviral Therapy \\ COR: Crude Odds Ratio \\ HIV: Human Immunodeficiency Virus \\ SPSS: Statistical Package for Social Sciences \\ WHO: World Health Organization
}

\section{ACKNOWLEDGEMENTS}

The authors acknowledge Federal Ministry of Education and Haramaya University for their financial support and Harari Regional Health Office and Dire Dawa City Administration Health Office for their cooperation and assistance during the entire process of the study.

\section{REFERENCES}

[1] United Nations Joint Program for HIV/AIDS (UNAIDS), "Nutrition, Food Security and HIV," Global Nutrition Report, Multi-Sectoral Nutrition Strategy Technical Brief, 2015.

[2] Piwoz EG, Preble EA, "HIV/AIDS and nutrition, "A review of the literature and recommendations for nutritional care and support in Sub-Saharan Africa," Support for Analysis and Research in Africa (SARA) Project, 2005.

[3] World Health Organization, Geneva, "Nutrient requirements for people living with HIV/AIDS," report of a technical consultation 13-15 May, 2003.

[4] Thapa R, Amatya A, Pahari DP, Bam K, Newman MS, "Nutritional status and its association with quality of life among people living with HIV attending public anti-retroviral therapy sites of Kathmandu Valley, Nepal," AIDS research and therapy, vol. 12, no. 1, pp. 14, 2015.

[5] Audain KA, Zotor FB, Amuna P, Ellahi B, "Food supplementation among HIV-infected adults in Sub-Saharan Africa, impact on treatment adherence and weight gain," Proceedings of the Nutrition Society, vol. 74, no. 4, pp. 517-525, 2015 
[6] Colecraft E., "HIV/AIDS, nutritional implications and impact on human development," Proceedings of the Nutrition Society, vol. 67, no. 1, pp. 109-13, 2008.

[7] Berhe N, Tegabu D, Alemayehu M, "Effect of nutritional factors on adherence to antiretroviral therapy among HIV-infected adults, a case control study in Northern Ethiopia," BMC infectious diseases, vol. 13, no. 1, pp. 233, 2013.

[8] Kendall T, Danel I, Cooper D et al., "Eliminating preventable HIV-related maternal mortality in sub-Saharan Africa: what do we need to know?," Journal of acquired immune deficiency syndromes, vol. 67, no. 4, pp. 250-258, 2014.

[9] Weiser S, Kimberly A, Eirikka K et al., "The association between food insecurity and mortality among HIV-infected individuals on HAART," J Acquir Immune Defic Syndr, vol. 52, no. 3, pp. 342-349, 2009.

[10] USAID, "Nutrition assessment, counselling and support for adolescents and adults living with HIV,” 2014.

[11] Kalofonos IA, "All I eat is ARVs": the paradox of AIDS treatment interventions in central Mozambique," Medical anthropology quarterly, vol. 24, no. 3, pp. 363-80, 2010.

[12] Talam NC, Rotich J, Kimaiyo S., "Factors affecting antimicrobial drug adherence among HIV/AIDS adult patients attending HIV/AIDS clinic at Moil Teaching and Referral Hospital, Eldoret, Kenya," East Afr J. Public Health, vol. 5, no. 2, pp. 74-8, 2008.

[13] Semba RD, Darnton-Hill I, de Pee S., "Addressing tuberculosis in the context of malnutrition and HIV co-infection," Food \& Nutrition Bulletin, vol. 31, no. 4, pp. S345-S364, 2010.

[14] Friis H., "Micronutrient interventions and HIV infection: a review of current evidence," Tropical medicine and international health, vol. 11, no. 12, pp. 1849-57, 2007.

[15] Tsasis P., "Adherence assessment to highly active antiretroviral therapy," AIDS Patient Care and STDs, vol. 15, no. 3, pp. 109-15. 2001.

[16] Burgin J NS, Dalrymple N., "The nutritional status of clinic attendees living with HIV/AIDS in St. Vicente and Grenadines," West Indian Med J., vol. 57, no. 5, pp. 438-43, 2008.

[17] Ludy MJ, Hendricks K, Houser R et al., "Body composition in adults infected with human immune deficiency virus in Khon Kaen, Thailand," Am J Trop Med Hyg, vol. 73, no. 4, pp. 815-9, 2005.

[18] World Health organization (WHO), "Indicators for Assessing Infant and Young Child Feeding Practices. Part 1: Definitions, Geneva," WHO Press, 2008.

[19] USAIDS Report on the global AIDS epidemic, 2012.

[20] Health, Ethiopia Ministry, "HSTP Health Sector Transformation Plan 2015/16-2019/20,” August.2015.

[21] Federal HIV/AIDS Prevention and Control Office Federal Ministry of Health, "Guidelines for Pediatric HIV/AIDS Care and Treatment in Ethiopia," 2007.

[22] Ethiopia Mini Demographic and Health Survey Indicators-FMOH, 2019.

[23] Francis Lwanga, Rhoda K. Wanyenze, Joseph KB Matovu, Teddy Chimulwa, and Christopher G. Orach. "Nutritional Status of HIV-infected Adolescents Enrolled into an HIV-care Program in Urban and Rural Uganda: A Cross-sectional Study," Journal of Nutrition and Health, vol. 3, no. 2, pp. 29-34, 2015.

[24] Berihun Megabiaw Zeleke, Belaynew Wasse Taye and Nikki Rogers, "Malnutrition among HIV Positive Children at two Referral Hospitals in Northwest Ethiopia," Official Journal of College of Medicine and Health Sciences, University of Gondar, Ethiopia, pp. 7-9, 2012.

[25] NHANES (National Health and Nutrition Examination Survey), Anthropometry procedures manual, 2007.

[26] Kennedy, G., Ballard, T., and Dop, M. C., Guidelines for Measuring Household and Individual Dietary Diversity. Nutrition and Consumer Protection Division 2013, Food and Agriculture Organization of the United Nations, 2010.

[27] Guidelines for measuring household and individual dietary diversity- FAO (Food and Agriculture Organization), 2013.

[28] Coates, J., Swindale, A., and Blinisky, P., Household Food Insecurity Access Scale (HFIAS) for Measurement of Food Access: Indicator Guide, VERSION 3 Washington, D.C.: Food and Nutrition Technical Assistance Project, Academy for Educational Development, 2007.

[29] FMOH (Federal Ministry of Health) Federal HIV/AIDS Prevention and Control Office Federal Ministry of Health. Guidelines for Pediatric HIV/AIDS Care and Treatment in Ethiopia. 2008.

[30] Haileselassie, B., Roba, K.T., and Woldegebreal, F., "Undernutrition and its Associated Factors among Pediatric Age Children Attending Antiretroviral Therapy in Eastern Ethiopia," East African Journal of Health and Biomedical Sciences, vol. 3, no. 1, pp. 1-12, 2019.

[31] Akintan, P.E., Akinsulie, A., Temiye, E., Esezobor, C., "Prevalence of Wasting, Stunting, and Underweight among HIV Infected Under-fives, in Lagos Using W.H.O Z score," Nigerian Quarterly Journal of Hospital Medicine, vol. 25, no. 2, pp.124-128, 2015.

[32] Nalwoga, A., et al., "Nutritional status of children living in a community with high HIV prevalence in rural Uganda: a cross-sectional population-based survey," Tropical medicine and international Health, vol. 15, no. 4, pp. 414-422, 2010.

[33] Pedrini, M., et al., "Clinical, nutritional and immunological characteristics of HIV-infected children in an area of high HIV prevalence," Journal of Tropical Pediatrics, vol. 61, no. 4, pp. 286-294, 2015.

[34] Tariku EZ, Abebe GA, Melketsedik ZA, Gutema BT., "Prevalence and factors associated with stunting and thinness among school age children in Arba Minch Health and Demographic Surveillance Site, Southern Ethiopia," PLOS ONE, vol. 13, no. 11, 2018. 